\title{
Cartesian composition and the problem of generalizing the MAC condition to quasi-multiautomata
}

\author{
Jan Chvalina, Štěpán Křehlík and Michal Novák
}

\begin{abstract}
When we assume that the input-set of an automaton without output is a semihypergroup instead of a monoid, we talk about quasi-multiautomata. Even though cartesian composition of quasi-automata is a commonly used concept, the cartesian composition of quasi-multiautomata has not been successfully constructed yet. In our paper we show that the straightforward transfer of the definition into the multivariate context fails. We suggest two possible solutions of this problem.
\end{abstract}

\section{Introduction}

In the algebraic theory of automata, various types of automata have been described and studied. One of these are quasi-automata, i.e. automata without output, structures consisting of the input-set (a monoid), state-set and a transition function. With the recent development of the hyperstructure theory, various generalizations of this concept have occurred. E.g. in the information technology, automata with a multivariate transition function are often used to describe various aspects of formal languages. If we permit the multivariate aspect in the input-set of quasi-automata, i.e. assume that the input-set is

Key Words: Cartesian composition of quasi-automata, Generalization of quasi-automata, Hyperstructure theory and automata, Quasi-multiautomata.

2010 Mathematics Subject Classification: 20N20; 20M35; 68Q70.

Received: 10.07 .2015

Revised: 02.09 .2015

Accepted: 15.12.2015 
a semihypergroup instead of a monoid, we arrive at the concept of a quasimultiautomaton. First steps in incorporating the hyperstruture theory into the concept of a quasi-automaton were done by Ashrafi and Madanshekaf [1] and Massouros and Mittas [11, 12]. This line of research was followed e.g. by Zhan, Mousavi and Jafarpour [17]. Also Chvalina proposed and with Chvalinová, Hošková or Dehghan Nezhad studied $[3,4,5,6]$ generalization of the concept. In papers such as $[3,4,5,6]$ the $G M A C$ condition, i.e. a condition which the transition function must fulfil in quasi-multiautomata, is studied. Relation of these two approaches is described in [3], Concluding remark. Chvalina's ideas have recently been transferred into the area of fuzzy (hyper)structures by Borzooei, Varasteh and Hasankhani [2].

When considering various operations with (quasi-)automata, concepts such as homogeneous or heterogeneous product or cartesian composition become relevant. In our paper we focus on the concept of cartesian composition, which was, for quasi-automata, introduced and studied by Dörfler [8] and which is often used in relation with fuzzy automata $[10,13,16]$.

We show that the GMAC condition, which distinguishes the transition function of a quasi-multiautomaton from a transition function of a quasiautomaton, cannot be applied straightforwadly for the cartesian composition of quasi-multiautomata. We suggest two extensions of this condition and show various aspects of their use and application. When constructing specific quasimultiautomata we expand results of $[3,4,5,6]$ and make use of hyperstructures and sets of differential operators motivated by functions used to model specific time processes in electrical engineering.

\section{Preliminaries}

\subsection{Quasi-automata and the cartesian composition}

Before introducing the concept of a quasi-multiautomaton recall the definition of a quasi-automaton.

Definition 1. By a quasi-automaton we mean a structure $\mathbb{A}=(I, S, \delta)$ such that $I \neq \emptyset$ is a monoid, $S \neq \emptyset$ and $\delta: I \times S \rightarrow S$ satisfies the following condition:

1) $\delta(e, s)=s$ for any state $s \in S$

2) $\delta(y, \delta(x, s))=\delta(x y, s)$ for any pair $x, y \in I$ and any state $s \in S$.

The set I is called the input-set, the set $S$ is called the state set and the mapping $\delta$ is called transition function. Condition 1 is called the unit condition (UC) while condition 2 is called the Mixed Associativity Condition (MAC). 
For quasi-automata there exist various types of products or compositions. In this paper we study the cartesian composition. Below we rewrite [8], Definition 4, into the form corresponding to definitions contained in [9].

Definition 2. Let $\mathbb{A}=\left(I, S, \delta_{\mathbb{A}}\right)$ and $\mathbb{B}=\left(J, T, \delta_{\mathbb{B}}\right)$ be two quasi-automata $\delta_{\mathbb{A}}: I \times S \rightarrow S, \delta_{\mathbb{B}}: J \times T \rightarrow T$ with disjoint input-sets $I, J$. By $\mathbb{A} \cdot \mathbb{B}$ we denote the automaton $\mathbb{A} \cdot \mathbb{B}=\left(I \cup J, S \times T, \delta_{\mathbb{A}} \cdot \delta_{\mathbb{B}}\right)$, where $\delta_{\mathbb{A}} \cdot \delta_{\mathbb{B}}:(I \cup J) \times(S \times T) \rightarrow S \times T$ is defined by

$$
\left(\delta_{\mathbb{A}} \cdot \delta_{\mathbb{B}}\right)(x,(s, t))= \begin{cases}\left(\delta_{\mathbb{A}}(x, s), t\right) & \text { if } x \in I, \\ \left(s, \delta_{\mathbb{B}}(x, t)\right) & \text { if } x \in J,\end{cases}
$$

for all $x \in I \cup J, s \in S$ and $t \in T$. The automaton $\mathbb{A} \cdot \mathbb{B}$ is called the cartesian composition of $\mathbb{A}$ and $\mathbb{B}$.

\subsection{The extension to quasi-multiautomata}

If in quasi-automata we suppose that the input-set $I$ is a semihypergroup instead of a monoid, we arrive at the concept of a quasi-multiautomaton. When defining it caution must be exercised when adjusting the conditions imposed on the transition function $\delta$. In the following definition, condition (1) is called GMAC, which stands for "Generalised Mixed Associativity Condition". For details regarding this condition see e.g. $[3,4,5,6]$. Notice that a semihypergroup is an associative hypergroupoid. For a deeper insight in the hyperstructure theory see e.g. [7].

Definition 3. A quasi-multiautomaton is a triad $\mathbb{A}=(I, S, \delta)$, where $(I, \cdot)$ is a semihypergroup, $S$ is a non-empty set and $\delta: I \times S \rightarrow S$ is a transition map satisfying the condition:

$$
\delta(b, \delta(a, s)) \in \delta(a \cdot b, s) \text { for all } a, b \in I, s \in S .
$$

The hyperstructure $(I, \cdot)$ is called the input semihypergroup of the quasi-multiautomaton $\mathbb{A}$, the set $S$ is called the state-set of the quasi-multiautomaton $\mathbb{A}$, and $\delta$ is called the transition function. Elements of the set $S$ are called states, elements of the set I are called input symbols (or words).

It can be shown that the wording of the GMAC condition (1) is suitable for the construction of both the homogeneous product and the heterogeneous product of quasi-multiautomata. However, the attempts to do the same for the cartesian composition fail. For details see Example 1, which is included after we specify the necessary input- and state-sets in Section 4.

In order to overcome this problem we construct two modifications of the GMAC condition (1) called E-GMAC and SE-GMAC, where "E" stands for 
"extended" and "SE" for "small extended". Further on we, for a given set $I$ and state $s$, by $\delta(I, s)$ mean the set $\{\delta(a, s)$; for all $a \in I\}$. In Definition 4 notice that $\delta(x \cdot y, s) \subseteq \delta(I, s)$. However, in Definition 6, in (6), there need not always be $(\delta \cdot \sigma)(x \diamond y,(s, t)) \subseteq\{\delta(I, s) \times \sigma(J, t)\}$.

Definition 4. If in Definition 3 we change condition (1) to

$$
\delta(y, \delta(x, s)) \in \delta(x \cdot y, s) \cup \delta(I, s) \text { for all } x, y \in I, s \in S,
$$

we call this condition E-GMAC and the multiautomaton $\mathbb{A}$ e-quasi-multiautomaton.

Traditionally, the concept of multiautomata need not include the "zero state" which may - yet need not - cause problems in some related considerations. However, zero states are very relevant in our context as their presence does influence our considerations - see e.g. proof of Theorem 6, part "ad 1)". Hence the following definition.

Definition 5. If in Definition 3 we change the state set to $S \cup\{0\}$ and condition (1) to

$$
\delta(y, \delta(x, s)) \in \delta(x \cdot y, s) \cup\{\delta(x, s), \delta(y, s)\} \text { for all } x, y \in I, s \in S,
$$

we call this condition SE-GMAC and the multiautomaton $\mathbb{A}$ se-quasi-multiautomaton.

Validity of the following lemma is obvious.

Lemma 1. Every quasi-multiautomaton is an e-quasi-multiautomaton. When its state set is extended with the "zero state", it is a se-quasi-multiautomaton as well. Similarly, validity of the GMAC condition (1) implies validity of both the E-GMAC condition (2) and (after the extension of the state set with the "zero state") SE-GMAC condition (3).

\section{The concept of the cartesian composition of quasi- multiautomata}

Now, using the E-GMAC and SE-GMAC conditions, we define the cartesian composition of quasi-multiautomata.

Definition 6. Let $\mathbb{A}=(I, S, \delta), \mathbb{B}=(J, T, \sigma)$ be e-quasi-multiautomata with input semihypergroups $I, J$ and transition maps $\delta: I \times S \rightarrow S, \sigma: J \times T \rightarrow T$ satisfying conditions

$$
\begin{aligned}
& \delta(y, \delta(x, s)) \in \delta(x \cdot y, s) \cup \delta(I, s) \text { for all } x, y \in I, s \in S, \\
& \sigma(y, \sigma(x, t)) \in \sigma(x \cdot y, t) \cup \sigma(J, t) \text { for all } x, y \in I, t \in T .
\end{aligned}
$$


By the cartesian composition of e-quasi-multiautomata $\mathbb{A}$ and $\mathbb{B}$, denoted as $\mathbb{A} \cdot{ }_{E} \mathbb{B}$, we mean the e-quasi-multiautomaton $\mathbb{A} \cdot{ }_{E} \mathbb{B}=(((I \cup J), \diamond), S \times T, \delta \cdot \sigma)$, where $\delta \cdot \sigma$ is for all $x \in I \cup J, s \in S$ and $t \in T$ defined by

$$
(\delta \cdot \sigma)(x,(s, t))= \begin{cases}(\delta(x, s), t) & \text { if } x \in I \\ (s, \sigma(x, t)) & \text { if } x \in J\end{cases}
$$

and $\diamond:(I \cup J) \times(I \cup J) \rightarrow \mathcal{P}^{*}(I \cup J)$ is for all $x, y \in I \cup J$ defined by

$$
x \diamond y= \begin{cases}x y \subseteq I & \text { if } x, y \in I, \\ x y \subseteq J & \text { if } x, y \in J, \\ \{x, y\} & \text { if } x \in I, y \in J \text { or } x \in J, y \in I\end{cases}
$$

and $\delta \cdot \sigma:(I \cup J) \times(S \times T) \rightarrow(S \times T)$ satisfies the condition:

$$
(\delta \cdot \sigma)(y,(\delta \cdot \sigma)(x,(s, t))) \in(\delta \cdot \sigma)(x \diamond y,(s, t)) \cup\{\delta(I, s) \times \sigma(J, t)\} .
$$

Definition 7. If in Definition 6 we consider two se-quasi-multiautomata and change condition (6) to

$(\delta \cdot \sigma)(y,(\delta \cdot \sigma)(x,(s, t))) \in(\delta \cdot \sigma)(x \diamond y,(s, t)) \cup\{(\delta(x, s), \sigma(y, t)),(\delta(y, s), \sigma(x, t))\}$,

we call the resulting se-quasi-multiautomaton the cartesian composition of sequasi-multiautomata $\mathbb{A}$ and $\mathbb{B}$ and denote is as $\mathbb{A} \cdot S E \mathbb{B}$.

Example 1 in Section 5 shows the flaws of the usual GMAC condition. One could easily show that using E-GMAC or SE-GMAC conditions removes the problem occurring there.

\section{State-sets and input-sets}

In order to develop results included in $[3,4,5,6]$ we now define specific inputsets and state-sets of quasi-multiautomata studied in these papers. However, notice that generalizing the reasoning concerning GMAC, E-GMAC and SEGMAC conditions is not based on the actual elements of the sets but on their properties. And below we do not impose any special assumptions.

First of all, we consider a commutative ring

$$
\mathscr{R}_{n}^{\mathbb{F}}(\Omega)=[\mathbb{F}(\Omega)]^{n}=\mathbb{F}(\Omega) \times \ldots \times \mathbb{F}(\Omega)
$$

formed by $n$-dimensional vectors $\left(f_{0}, \ldots, f_{n-1}\right)$ of real functions of $m$-variables $f: \Omega \rightarrow \mathbb{R}$, where $\emptyset \neq \Omega \subseteq \mathbb{R}^{m}, m \in \mathbb{N}$. The binary operation of addition 
is defined as usually for vectors, i.e. component-wise, while multiplication is defined by

$$
\left(f_{0}, \ldots, f_{n-1}\right) \cdot\left(g_{0}, \ldots, g_{n-1}\right)=\left(f_{0} g_{0}, \ldots, f_{n-1} g_{n-1}\right),
$$

for any pair of vectors $\vec{f}=\left(f_{0}, \ldots, f_{n-1}\right), \vec{g}=\left(g_{0}, \ldots, g_{n-1}\right) \in \mathscr{R}_{n}^{\mathbb{F}}(\Omega)$.

Second, for a positive integer $n \geq 2$ and an interval $T \subseteq \mathbb{R}$ denote by $\mathbb{L} \mathbb{A}_{n}(T)$ the set of all linear differential operators of the $n$-th order $L\left(p_{0}, \ldots, p_{n-1}\right)$, where $p_{k} \in C(T)$, with the action

$$
L\left(p_{0}, \ldots, p_{n-1}\right) y=y^{(n)}(x)+p_{n-1}(x) y^{(n-1)}(x)+\ldots+p_{0}(x) y(x)
$$

for any $y \in C^{n}(T)$.

Third, we consider the $n$-dimensional vector space over the field of real numbers

$$
\mathcal{R}_{n}=\left\{\left(s_{0}, \ldots, s_{n-1}\right) ; s_{k} \in \mathbb{R}, k=0, \ldots, n-1\right\},
$$

on which we define a binary hyperoperation "•" by

$$
\left(r_{0}, \ldots, r_{n-1}\right) \bullet\left(s_{0}, \ldots, s_{n-1}\right)=\left\{\left(t_{0}, \ldots, t_{n-1}\right), t_{k} \geq r_{k} s_{k}, k=0, \ldots, n-1\right\} .
$$

Lemma 2. The hypergroupoid $\left(\mathcal{R}_{n}, \bullet\right)$ is a commutative semihypergroup.

Proof. Suppose arbitrary $\left(r_{0}, \ldots, r_{n-1}\right)=\vec{r},\left(s_{0}, \ldots, s_{n-1}\right)=\vec{s},\left(t_{0}, \ldots, t_{n-1}\right)=$ $\vec{t} \in \mathcal{R}_{n}$. Then $\vec{r} \bullet(\vec{s} \bullet \vec{t})=\vec{r} \bullet\{\vec{u}, \vec{u} \geq \vec{s} \vec{t}\}=\bigcup_{\vec{u} \geq \vec{s} \vec{t}} \vec{r} \vec{u}=\{\vec{v} ; \vec{v} \geq \vec{r} \vec{s} \vec{t}\}=$ $\bigcup_{\vec{w}>\vec{r} \vec{s}} \vec{r} \vec{w}=(\vec{r} \bullet \vec{s}) \bullet \vec{t}$. Thus $\left(\mathcal{R}_{n}, \bullet\right)$ satisfies the associativity axiom and is a semihypergroup. Commutativity is obvious.

Fourth, denote $\mathbb{M}_{n, n}\left(\mathbb{R}^{+}\right)$the set of square matrices of order $n$ with nonnegative real entries. On $\mathbb{M}_{n, n}\left(\mathbb{R}^{+}\right)$regard such a relation $\leq$that for matrices $\mathbf{A}=\left(a_{i j}\right), \mathbf{B}=\left(b_{i j}\right)$ we write $\mathbf{A} \leq \mathbf{B}$ whenever $a_{i j} \leq b_{i j}$ for all $i, j \in\{1, \ldots, n\}$. Further on we denote $\mathbb{N}_{n}=\{1, \ldots, n\}$. We define the hyperoperation "o" on $\mathbb{M}_{n, n}\left(\mathbb{R}^{+}\right)$by:

$$
\mathbf{A} \circ \mathbf{B}=\left\{\mathbf{C} \in \mathbb{M}_{n, n}\left(\mathbb{R}^{+}\right) ; \min \{\mathbf{A}, \mathbf{B}\} \leq \mathbf{C}\right\}
$$

i.e.

$$
\begin{gathered}
{\left[\begin{array}{ccc}
a_{11} & \ldots & a_{1 n} \\
\ldots & \ldots & \ldots \\
a_{n 1} & \ldots & a_{n n}
\end{array}\right] \circ\left[\begin{array}{ccc}
b_{11} & \ldots & b_{1 n} \\
\ldots & \ldots & \ldots \\
b_{n 1} & \ldots & b_{n n}
\end{array}\right]=} \\
=\left\{\left[\begin{array}{ccc}
c_{11} & \ldots & c_{1 n} \\
\ldots & \ldots & \ldots \\
c_{n 1} & \ldots & c_{n n}
\end{array}\right] ; \min \left\{a_{i j}, b_{i j}\right\} \leq c_{i j}, i, j \in \mathbb{N}_{n}\right\},
\end{gathered}
$$


where $a_{i j}, b_{i j}, c_{i j} \in \mathbb{R}^{+}$. Further on when showing different aspects of the E-GMAC and SE-GMAC conditions we will also need to restrict our considerations on matrices, entries of which are not only non-negative but also greater than 1. Therefore we denote

$$
\mathbb{M}_{n, n}\left(\mathbb{R}_{1}^{+}\right)=\left\{\mathbf{M} \in \mathbb{M}_{n, n}\left(\mathbb{R}^{+}\right) ; \mathbf{M}=\left(m_{i j}\right), m_{i j} \geq 1, i, j \in \mathbb{N}_{n}\right\}
$$

Lemma 3. The hypergroupoids $\left(\mathbb{M}_{n, n}\left(\mathbb{R}^{+}\right)\right.$, o $)$and $\left(\mathbb{M}_{n, n}\left(\mathbb{R}_{1}^{+}\right)\right.$, o $)$are commutative semihypergroups.

Proof. The above defined minimum of square matrices is an associative and commutative operation on $\mathbb{M}_{n, n}\left(\mathbb{R}^{+}\right)$. Moreover, the above defined ordering of matrices is reflexive and transitive. Finally, $\left(\mathbb{M}_{n, n}\left(\mathbb{R}^{+}\right), \min , \leq\right)$ is obviously a quasi-ordered semigroup. Thus, $\left(\mathbb{M}_{n, n}\left(\mathbb{R}^{+}\right), \circ\right)$ is a commutative $E L-$ semihypergroup in the sense of $[14,15]$. The fact that $\left(\mathbb{M}_{n, n}\left(\mathbb{R}_{1}^{+}\right)\right.$, o) is a semihypergroup is in this respect obvious.

Remark 1. In fact, it can be proved that hyperstructures $\left(\mathcal{R}_{n}, \bullet\right),\left(\mathbb{M}_{n, n}\left(\mathbb{R}^{+}\right), \circ\right)$ and $\left(\mathbb{M}_{n, n}\left(\mathbb{R}_{1}^{+}\right), \circ\right)$ are hypergroups. However, this is not needed for our considerations. Also notice that since we aim at constructing the cartesian composition of quasi-multiautomata, the fact that structures $\left(\mathbb{M}_{n, n}\left(\mathbb{R}^{+}\right), \circ\right)$ and $\left(\mathcal{R}_{n}, \bullet\right)$ are disjoint for $n \geq 2$ is important.

For the purpose of the following theorem we define a binary hyperoperation

$$
\triangle:\left(\mathcal{R}_{n} \cup \mathbb{M}_{n, n}\left(\mathbb{R}^{+}\right)\right) \times\left(\mathcal{R}_{n} \cup \mathbb{M}_{n, n}\left(\mathbb{R}^{+}\right)\right) \rightarrow \mathcal{P}^{*}\left(\mathcal{R}_{n} \cup \mathbb{M}_{n, n}\left(\mathbb{R}^{+}\right)\right)
$$

by the following rule for arbitrary $X, Y \in \mathcal{R}_{n} \cup \mathbb{M}_{n, n}\left(\mathbb{R}^{+}\right)$:

1. If $X=\vec{r} \in \mathcal{R}_{n}, Y=\vec{s} \in \mathcal{R}_{n}$, then we put $X \triangle Y=\vec{r} \bullet \vec{s}$.

2. If $X=\mathbf{A} \in \mathbb{M}_{n, n}\left(\mathbb{R}^{+}\right), Y=\mathbf{B} \in \mathbb{M}_{n, n}\left(\mathbb{R}^{+}\right)$, then we put $X \triangle Y=\mathbf{A} \circ \mathbf{B}$.

3. If $X=\vec{r} \in \mathcal{R}_{n}, Y=\mathbf{A} \in \mathbb{M}_{n, n}\left(\mathbb{R}^{+}\right)$, then we put $X \triangle Y=\{X, Y\}=$ $\{\vec{r}, \mathbf{A}\}=\mathbf{A} \triangle \vec{r}=Y \triangle X$.

Theorem 1. The hypergroupoid $\left(\left(\mathcal{R}_{n} \cup \mathbb{M}_{n, n}\left(\mathbb{R}^{+}\right)\right), \triangle\right)$, where the hyperoperation " $\triangle$ " is defined by (15), is a commutative semihypergroup.

Proof. When supposing that $X, Y \in\left(\mathcal{R}_{n} \cup \mathbb{M}_{n, n}\left(\mathbb{R}^{+}\right)\right)$we have to consider the following three cases:

1) $X=\vec{r} \in \mathcal{R}_{n}, Y=\vec{s} \in \mathcal{R}_{n}$,

2) $X=\mathbf{A} \in \mathbb{M}_{n, n}\left(\mathbb{R}^{+}\right), Y=\mathbf{B} \in \mathbb{M}_{n, n}\left(\mathbb{R}^{+}\right)$, 
3) $X=\vec{r} \in \mathcal{R}_{n}, Y=\mathbf{A} \in \mathbb{M}_{n, n}\left(\mathbb{R}^{+}\right)$.

The first case is dealt with by Lemma 2. The second case is dealt with by Lemma 3. Thus we have to verify associativity in the third case only. There we suppose $X, Y, Z \in\left(\mathcal{R}_{n} \cup \mathbb{M}_{n, n}\left(\mathbb{R}^{+}\right)\right)$arbitrary. Then

$$
\begin{gathered}
(X \triangle Y) \triangle Z=\{X, Y\} \triangle Z=\{(X \triangle Z)\} \cup\{(Y \triangle Z)\}= \\
=\{X, Z\} \cup\{Y, Z\}=\{X, Y, Z\}=\{X, Y\} \cup\{X, Z\}= \\
=\{(X \triangle Y)\} \cup\{(X \triangle Z)\}=X \triangle\{Y, Z\}=X \triangle(Y \triangle Z) .
\end{gathered}
$$

Thus the hyperoperation " $\triangle$ " is associative. Its commutativity is obvious.

\section{Quasi-multiautomata and insufficiency of the GMAC condition for their cartesian composition}

In this section we construct two quasi-multiautomata, which are, thanks to Lemma 1, also e-quasi-multiautomata. One of these will have the input semihypergroup $\left(\mathcal{R}_{n}, \bullet\right)$ and state-set $\mathbb{L} \mathbb{A}_{n}(T)$ while the other will have the input semihypergroup $\left(\mathbb{M}_{n, n}\left(\mathbb{R}_{1}^{+}\right), \circ\right)$ and the state-set $\mathscr{R}_{n}^{\mathbb{F}}(\Omega)$. We are also going to construct an e-quasi-multiautomaton with the input semihypergroup $\left(\mathbb{M}_{n, n}\left(\mathbb{R}^{+}\right), \circ\right)$ and the state-set $\mathscr{R}_{n}^{\mathbb{F}}(\Omega)$. This can be treated as an example of a structure which is an e-quasi-multiautomaton yet not a quasi-multiautomaton. Example 2 gives an idea of consequences of this fact for the cartesian composition in a specific context of $n=2$.

Theorem 2. The structure $\mathbb{A}_{1}=\left(\left(\mathcal{R}_{n}, \bullet\right), \mathbb{L}_{n}(T), \delta_{1}\right)$, where the transition function $\delta_{1}: \mathcal{R}_{n} \times \mathbb{L} \mathbb{A}_{n}(T) \rightarrow \mathbb{L} \mathbb{A}_{n}(T)$ is defined by

$$
\delta_{1}(\vec{r}, L(\vec{p}))=(\vec{r} \cdot L(\vec{p}))=L\left(r_{0} p_{0}, \ldots, r_{n-1} p_{n-1}\right)
$$

for all $\vec{r} \in \mathcal{R}_{n}$ and $L(\vec{p}) \in \mathbb{L} \mathbb{A}_{n}(T)$, is a quasi-multiautomaton.

Proof. Thanks to Lemma 2 we already know that $\left(\mathcal{R}_{n}, \bullet\right)$ is a semihypergroup. Next, we show that the structure $\mathbb{A}_{1}$ satisfies the GMAC condition (1), i.e. $\delta_{1}\left(\vec{r}, \delta_{1}(\vec{s}, L(\vec{p}))\right) \in \delta_{1}(\vec{r} \bullet \vec{s}, L(\vec{p}))$. The calculation of the left hand side:

$$
\delta_{1}\left(\vec{r}, \delta_{1}(\vec{s}, L(\vec{p}))\right)=\delta_{1}\left(\vec{r}, L\left(s_{0} p_{0}, \ldots, s_{n-1} p_{n-1}\right)\right)=L\left(r_{0} s_{0} p_{0}, \ldots, r_{n-1} s_{n-1} p_{n-1}\right) .
$$

The calculation of the right hand side:

$$
\delta_{1}(\vec{r} \bullet \vec{s}, L(\vec{p}))=\delta_{1}\left(\bigcup_{\vec{t} \geq \vec{r} \vec{s}}(\vec{t}, L(\vec{p}))\right)=\left\{L\left(t_{0} p_{0}, \ldots, t_{n-1} p_{n-1}\right) ; \vec{t} \in \mathcal{R}_{n}, \vec{t} \geq \vec{r} \vec{s}\right\} .
$$


For $\vec{t}=\vec{r} \vec{s}$ we have $L\left(r_{0} s_{0} p_{0}, \ldots, r_{n-1} s_{n-1} p_{n-1}\right)=L\left(t_{0} p_{0}, \ldots, t_{n-1} p_{n-1}\right) ; \vec{t} \in$ $\mathcal{R}_{n}$. Thus $L\left(r_{0} s_{0} p_{0}, \ldots, r_{n-1} s_{n-1} p_{n-1}\right) \in \delta_{1}(\vec{r} \bullet \vec{s}, L(\vec{p}))$ and $\mathbb{A}_{1}$ is a quasimultiautomaton.

Theorem 3. The structure $\mathbb{A}_{2}=\left(\left(\mathbb{M}_{n, n}\left(\mathbb{R}_{1}^{+}\right), \circ\right), \mathscr{R}_{n}^{\mathbb{F}}(\Omega), \delta_{2}\right)$, where the transition function $\delta_{2}:\left(\mathbb{M}_{n, n}\left(\mathbb{R}_{1}^{+}\right), \circ\right) \times \mathscr{R}_{n}^{\mathbb{F}}(\Omega) \rightarrow \mathscr{R}_{n}^{\mathbb{F}}(\Omega)$ is defined by $\delta_{2}(\mathbf{A}, \vec{p})=$ $\vec{p} \cdot \mathbf{A}=\vec{q}$, where

$$
\vec{p} \cdot \mathbf{A}=\left(p_{0}, \ldots, p_{n-1}\right) \cdot\left[\begin{array}{ccc}
a_{11} & \ldots & a_{1 n} \\
\ldots & \ldots & \ldots \\
a_{n 1} & \ldots & a_{n n}
\end{array}\right]=\left(q_{0}, \ldots, q_{n-1}\right)=\vec{q}
$$

for all $\mathbf{A} \in \mathbb{M}_{n, n}\left(\mathbb{R}_{1}^{+}\right)$and $\vec{p} \in \mathscr{R}_{n}^{\mathbb{F}}(\Omega)$, is a quasi-multiautomaton.

Proof. Thanks to Lemma 3 we already know that $\left(\mathbb{M}_{n, n}\left(\mathbb{R}_{1}^{+}\right), \circ\right)$ is a semihypergroup. We show that $\operatorname{GMAC}(1)$, i.e. $\delta_{2}\left(\mathbf{B}, \delta_{2}(\mathbf{A}, \vec{p})\right) \in \delta_{2}(\mathbf{A} \circ \mathbf{B}, \vec{p})$, holds in $\mathbb{A}_{2}$. The calculation of the left-hand side:

$$
\begin{gathered}
\delta_{2}\left(\left(b_{i j}\right), \delta_{2}\left(\left(a_{i j}\right),\left(p_{0}, \ldots, p_{n-1}\right)\right)\right)=\delta_{2}\left(\left(b_{i j}\right),\left(p_{0}, \ldots, p_{n-1}\right) \cdot\left[\begin{array}{lll}
a_{11} & \ldots & a_{1 n} \\
\ldots & \ldots & \ldots \\
a_{n 1} & \ldots & a_{n n}
\end{array}\right]\right)= \\
=\delta_{2}\left(\left(b_{i j}\right),\left(\sum_{i=1}^{n} a_{i 1} p_{i-1}, \ldots, \sum_{i=1}^{n} a_{i n} p_{i-1}\right)\right)= \\
=\left(\sum_{i=1}^{n} a_{i 1} p_{i-1}, \ldots, \sum_{i=1}^{n} a_{i n} p_{i-1}\right) \cdot\left[\begin{array}{lll}
b_{11} & \ldots & b_{1 n} \\
\ldots & \ldots & \ldots \\
b_{n 1} & \ldots & b_{n n}
\end{array}\right]= \\
=\left(b_{11} \sum_{i=1}^{n} a_{i 1} p_{i-1}+\ldots+b_{n 1} \sum_{i=1}^{n} a_{i n} p_{i-1}, \ldots,\right. \\
=\left(\sum_{i, j=1}^{n} a_{i j} b_{j 1} p_{j-1}, \ldots, \sum_{i, j=1}^{n} a_{i j} b_{j n} p_{j-1}\right)=\left(q_{0}, \ldots, q_{n-1}\right) .
\end{gathered}
$$

The calculation of the right-hand side:

$$
\begin{gathered}
\delta_{2}\left(\mathbf{A} \circ \mathbf{B},\left(p_{0}, \ldots, p_{n-1}\right)\right)= \\
=\left\{\left(p_{0}, \ldots, p_{n-1}\right) \cdot \mathbf{C} ; \mathbf{C}=\left(c_{i j}\right) ; c_{i j} \geq \min \left\{a_{i j}, b_{i j}\right\}, i, j \in \mathbb{N}_{n}\right\}=
\end{gathered}
$$




$$
\begin{aligned}
=\left\{\left(c_{11} p_{0}\right)+\ldots+c_{n 1} p_{n-1}, \ldots,\right. \\
\left.\left.\quad c_{1 n} p_{0}+\ldots+c_{n n} p_{n-1}\right) ; c_{i j} \geq \min \left\{a_{i j}, b_{i j}\right\}, i, j \in \mathbb{N}_{n}\right\} .
\end{aligned}
$$

Since we regard the set $\mathbb{M}_{n, n}\left(\mathbb{R}_{1}^{+}\right)$, we have in general $q_{k-1}=c_{1 k} p_{0}+\ldots+$ $c_{n k} p_{n-1}$. Since we have

$$
c_{j k}=\sum_{j, i=1}^{n} a_{i k} b_{j k} \geq \min \left\{a_{i k} b_{j k}\right\} \geq 1,
$$

we obtain

$$
\begin{gathered}
\left(q_{0}, \ldots, q_{n-1}\right)=\left(\sum_{i, j=1}^{n} a_{i j} b_{j 1} p_{j-1}, \ldots, \sum_{i, j=1}^{n} a_{i j} b_{j n} p_{j-1}\right) \in \\
\in\left\{\left(c_{11} p_{0}+\ldots+c_{n 1} p_{n-1}, \ldots,\right.\right. \\
\left.\left.c_{1 n} p_{0}+\ldots+c_{n n} p_{n-1}\right) ; c_{i j} \geq \min \left\{a_{i j}, b_{i j}\right\}, i, j \in \mathbb{N}_{n}\right\}= \\
=\delta_{2}\left(\mathbf{A} \circ \mathbf{B},\left(p_{0}, \ldots, p_{n-1}\right)\right) .
\end{gathered}
$$

Hence GMAC (1) is satisfied.

Theorem 4. If in Theorem 3 we regard $\left(\mathbb{M}_{n, n}\left(\mathbb{R}^{+}\right)\right.$, o) instead of $\left(\mathbb{M}_{n, n}\left(\mathbb{R}_{1}^{+}\right), \circ\right)$, then $\mathbb{A}_{3}=\left(\left(\mathbb{M}_{n, n}\left(\mathbb{R}^{+}\right), \circ\right), \mathscr{R}_{n}^{\mathbb{F}}(\Omega), \delta_{3}\right)$, where $\delta_{3} \equiv \delta_{2}$, is an e-quasi-multiautomaton.

Proof. The proof follows the pattern of the proof of Theorem 3 yet the set on the right-hand side is united with $\left\{\delta_{3}\left(\mathbb{M}_{n, n}\left(\mathbb{R}^{+}\right), \vec{p}\right)\right\}$, i.e. with

$$
\begin{gathered}
\left\{\delta_{3}\left(\left[\begin{array}{ccc}
m_{11} & \ldots & m_{1 n} \\
\ldots & \ldots & \ldots \\
m_{n 1} & \ldots & m_{n n}
\end{array}\right],\left(p_{0}, \ldots, p_{n-1}\right)\right) ; \text { for all } \mathbf{M}=\left(m_{i j}\right) \in \mathbb{M}_{n, n}\left(\mathbb{R}^{+}\right)\right\}= \\
=\left\{\sum_{i=1}^{n} m_{i 1} p_{i-1}, \ldots, \sum_{i=1}^{n} m_{i n} p_{i-1} ; m_{i j} \in \mathbb{R}^{+}\right\}
\end{gathered}
$$

If we realize that for an arbitrary pair of matrices $\mathbf{A}, \mathbf{B} \in \mathbb{M}_{n, n}\left(\mathbb{R}^{+}\right)$and an arbitrary vector $\vec{p} \in \mathscr{R}_{n}^{\mathbb{F}}(\Omega)$ there holds $(\vec{p} \cdot \mathbf{A}) \cdot \mathbf{B}=\vec{p} \cdot(\mathbf{A} \cdot \mathbf{B})$, we can denote every $k$-th sum $\sum_{i, j=1}^{n} a_{i j} b_{j k}$ on the left-hand side by $\sum_{i=1}^{n} m_{i 1}$ and validity of the inclusion requested for the E-GMAC condition becomes obvious. 
The following is an example showing that the plain GMAC condition

$$
(\delta \cdot \sigma)(y,(\delta \cdot \sigma)(x,(s, t))) \in(\delta \cdot \sigma)(x \triangle y,(s, t))
$$

is not sufficient to construct the cartesian composition of quasi-multiautomata $\mathbb{A}_{1}$ and $\mathbb{A}_{3}$. It can be easily shown that using either the E-GMAC condition (6) or the SE-GMAC condition (7) removes the problem. For the verification using SE-GMAC see Example 3.

Example 1. Suppose the structure $\mathbb{A}=\mathbb{A}_{1} \cdot \mathbb{A}_{2}$ for $n=2$ and specific inputs $X=(1,2), \mathbf{Y}=\left[\begin{array}{ll}2 & 4 \\ 3 & 1\end{array}\right]$, binary hyperoperation $\triangle$ defined by (15) and state $\left(L\left(x^{2}, x\right),(x, 0)\right)$, where - denotes the cartesian composition of quasimultiautomata $\mathbb{A}_{1}$ and $\mathbb{A}_{2}$ defined using Definition 6 with condition (16) instead of (6). Now,

$$
\begin{aligned}
\left(\delta_{1} \cdot \delta_{3}\right)\left((1,2),\left(\delta_{1} \cdot \delta_{3}\right)\right. & \left.\left(\left[\begin{array}{ll}
2 & 4 \\
3 & 1
\end{array}\right],\left(L\left(x^{2}, x\right),(x, 0)\right)\right)\right) \notin \\
& \notin\left(\delta_{1} \cdot \delta_{3}\right)\left((1,2) \triangle\left[\begin{array}{ll}
2 & 4 \\
3 & 1
\end{array}\right],\left(L\left(x^{2}, x\right),(x, 0)\right)\right)
\end{aligned}
$$

because

$$
\begin{aligned}
\delta_{1}\left((1,2),\left(L\left(x^{2}, x\right), \delta_{3}\left(\left[\begin{array}{ll}
2 & 4 \\
3 & 1
\end{array}\right],(x, 0)\right)\right) \notin\right. & \\
& \notin\left(\delta_{1} \cdot \delta_{3}\right)\left((1,2) \triangle\left[\begin{array}{ll}
2 & 4 \\
3 & 1
\end{array}\right],\left(L\left(x^{2}, x\right),(x, 0)\right)\right)
\end{aligned}
$$

i.e.

$$
\begin{gathered}
\delta_{1}\left((1,2),\left(L\left(x^{2}, x\right),(2 x, 4 x)\right) \notin\left(\delta_{1} \cdot \delta_{3}\right)\left(\left\{(1,2),\left[\begin{array}{ll}
2 & 4 \\
3 & 1
\end{array}\right]\right\},\left(L\left(x^{2}, x\right),(x, 0)\right)\right)\right. \\
\left(L\left(x^{2}, 2 x\right),(2 x, 4 x)\right) \notin\left\{\delta_{1}\left((1,2), L\left(x^{2}, x\right)\right)\right\} \cup\left\{\delta_{3}\left(\left[\begin{array}{ll}
2 & 4 \\
3 & 1
\end{array}\right],(x, 0)\right)\right\} \\
\left(L\left(x^{2}, 2 x\right),(2 x, 4 x)\right) \notin\left\{\left(L\left(x^{2}, 2 x\right),(x, 0)\right),\left(L\left(x^{2}, x\right),(2 x, 4 x)\right)\right\} .
\end{gathered}
$$

On the left-hand side there is an element consisting of two pairs yet this element is not included in the right-hand side. Thus the plain GMAC condition without the extensions provided by E-GMAC (6) and SE-GMAC (7) does not hold. 


\section{Cartesian composition using E-GMAC and SE-GMAC}

In the following theorem and in Theorem 6 recall the swapping of components A and $\vec{p}$ in the definition of the transition function $\delta_{2}$ included in Theorem 3 (and $\delta_{3}$ included in Theorem 4 ), which is caused by the necessity to multiply a vector and a matrix.

Theorem 5. Consider e-quasi-multiautomata $\mathbb{A}_{1}$ and $\mathbb{A}_{3}$ constructed using Theorem 2 and Theorem 4. Define

$$
\left(\delta_{1} \cdot \delta_{3}\right):\left(\mathcal{R}_{n} \cup \mathbb{M}_{n, n}\left(\mathbb{R}^{+}\right)\right) \times\left(\mathbb{L}_{n}(T) \times \mathscr{R}_{n}^{\mathbb{F}}(\Omega)\right) \rightarrow \mathbb{L} \mathbb{A}_{n}(T) \times \mathscr{R}_{n}^{\mathbb{F}}(\Omega)
$$

by

$$
\left(\delta_{1} \cdot \delta_{3}\right)(X,(L(\vec{p}), \vec{q}))= \begin{cases}\left(\delta_{1}(X, L(\vec{p})), \vec{q}\right) & \text { if } X=\vec{r} \in \mathcal{R}_{n} \\ \left(L(\vec{p}), \delta_{3}(X, \vec{q})\right) & \text { if } X=\mathbf{A} \in \mathbb{M}_{n, n}\left(\mathbb{R}^{+}\right) .\end{cases}
$$

Then the structure $\left(\left(\left(\mathcal{R}_{n} \cup \mathbb{M}_{n, n}\left(\mathbb{R}^{+}\right)\right), \triangle\right), \mathbb{L}_{n}(T) \times \mathscr{R}_{n}^{\mathbb{F}}(\Omega), \delta_{1} \cdot \delta_{3}\right)$ is the cartesian composition $\mathbb{A}_{1} \cdot E \mathbb{A}_{3}$.

Proof. We are going to show that the structure $\left(\left(\left(\mathcal{R}_{n} \cup \mathbb{M}_{n, n}\left(\mathbb{R}^{+}\right)\right), \triangle\right), \mathbb{L} \mathbb{A}_{n}(T) \times\right.$ $\left.\mathscr{R}_{n}^{\mathbb{F}}(\Omega), \delta_{1} \cdot \delta_{3}\right)$ satisfies E-GMAG. We have four cases:

1) $X=\vec{r} \in \mathcal{R}_{n}, Y=\vec{s} \in \mathcal{R}_{n}$,

2) $X=\mathbf{A} \in \mathbb{M}_{n, n}\left(\mathbb{R}^{+}\right), Y=\mathbf{B} \in \mathbb{M}_{n, n}\left(\mathbb{R}^{+}\right)$,

3) $X=\vec{r} \in \mathcal{R}_{n}, Y=\mathbf{A} \in \mathbb{M}_{n, n}\left(\mathbb{R}^{+}\right)$,

4) $X=\mathbf{A} \in \mathbb{M}_{n, n}\left(\mathbb{R}^{+}\right), Y=\vec{r} \in \mathcal{R}_{n}$.

We show that the E-GMAG condition (6), i.e.

$$
\begin{aligned}
\left(\delta_{1} \cdot \delta_{3}\right)\left(X,\left(\delta_{1} \cdot \delta_{3}\right)(Y,(L(\vec{p}), \vec{q}))\right) & \in\left(\delta_{1} \cdot \delta_{3}\right)(X \triangle Y,(L(\vec{p}), \vec{q})) \cup \\
& \cup\left\{\delta_{1}\left(\mathcal{R}_{n}, L(\vec{p})\right) \times \delta_{3}\left(\mathbb{M}_{n, n}\left(\mathbb{R}^{+}\right), \vec{q}\right)\right\}
\end{aligned}
$$

is valid in all of them.

ad 1) In (19) we have: The calculation of the left hand side:

$$
\begin{aligned}
\left(\delta_{1} \cdot \delta_{3}\right)\left(X,\left(\delta_{1} \cdot \delta_{3}\right)(Y,(L(\vec{p}), \vec{q}))\right) & =\left(\delta_{1}\left(\vec{r},\left(\delta_{1}(\vec{s}, L(\vec{p})), \vec{q}\right)\right)\right)= \\
=\left(\delta_{1}(\vec{r},(L(\vec{s} \vec{p}), \vec{q}))\right) & =(L(\vec{r} \vec{s} \vec{p}), \vec{q}) .
\end{aligned}
$$


The calculation of the right hand side:

$$
\begin{aligned}
& \left(\delta_{1} \cdot \delta_{3}\right)(X \triangle Y,(L(\vec{p}), \vec{q})) \cup\left\{\delta_{1}\left(\mathcal{R}_{n}, L(\vec{p})\right) \times \delta_{3}\left(\mathbb{M}_{n, n}\left(\mathbb{R}^{+}\right), \vec{q}\right)\right\}= \\
& \quad=\delta_{1}(\vec{r} \bullet \vec{s},(L(\vec{p}), \vec{q})) \cup\left\{\delta_{1}\left(\mathcal{R}_{n}, L(\vec{p})\right) \times \delta_{3}\left(\mathbb{M}_{n, n}\left(\mathbb{R}^{+}\right), \vec{q}\right)\right\}= \\
& =\bigcup_{\vec{t} \geq \vec{r} \vec{s}}\left(\delta_{1}(\vec{t}, L(\vec{p})), \vec{q}\right) \cup\left\{\bigcup_{\vec{r} \in \mathcal{R}_{n}} \delta_{1}(\vec{r}, L(\vec{p})) \times \bigcup_{\mathbf{A} \in \mathbb{M}_{n, n}\left(\mathbb{R}^{+}\right)} \delta_{3}(\vec{q}, \mathbf{A})\right\}= \\
& =\{(L(\vec{t} \vec{p}), \vec{q}) ; \vec{t} \geq \vec{r} \vec{s}\} \cup\left\{(L(\vec{r} \vec{p}), \vec{\varphi}) ; \vec{r} \in \mathcal{R}_{n}, \vec{\varphi} \in \mathscr{R}_{n}^{\mathbb{F}}(\Omega)\right\} .
\end{aligned}
$$

For $\vec{t}=\vec{r} \vec{s}$ we have

$$
\left(L\left(r_{0} s_{0} p_{0}, \ldots, r_{n-1} s_{n-1} p_{n-1}\right), \vec{q}\right)=\left(L\left(t_{0} p_{0}, \ldots, t_{n-1} p_{n-1}\right), \vec{q}\right)
$$

where $\vec{t} \in \mathcal{R}_{n}$. Thus

$$
\begin{aligned}
\left(L\left(r_{0} s_{0} p_{0}, \ldots, r_{n-1} s_{n-1} p_{n-1}\right), \vec{q}\right) \in\{(L(\vec{t} \vec{p}), \vec{q}) ; \vec{t} \geq \vec{r} \vec{s}\} \cup \\
\left\{(L(\vec{r} \vec{p}), \vec{\varphi}) ; \vec{r} \in \mathcal{R}_{n}, \vec{\varphi} \in \mathscr{R}_{n}^{\mathbb{F}}(\Omega)\right\}
\end{aligned}
$$

and E-GMAC is satisfied.

ad 2) In (19) we have: The calculation of the left hand side:

$$
\begin{aligned}
& \delta_{3}\left(\mathbf{A},\left(L(\vec{p}), \delta_{3}(\mathbf{B}, \vec{q})\right)\right)=\delta_{3}\left(\mathbf{A},\left(L(\vec{p}),\left(q_{0}, \ldots, q_{n-1}\right) \cdot\left[\begin{array}{ccc}
b_{11} & \ldots & b_{1 n} \\
\ldots & \ldots & \ldots \\
b_{m 1} & \ldots & b_{m n}
\end{array}\right]\right)\right)= \\
& =\delta_{3}\left(\mathbf{A},\left(L(\vec{p}),\left(\sum_{i=1}^{n} b_{i 1} q_{i-1}, \ldots, \sum_{i=1}^{n} b_{i n} q_{i-1}\right)\right)\right)= \\
& \left.=\left(L(\vec{p}), \delta_{3}\left(\mathbf{A},\left(\sum_{i=1}^{n} b_{i 1} q_{i-1}, \ldots, \sum_{i=1}^{n} b_{i n} q_{i-1}\right)\right)\right)\right)= \\
& \left.=\left(L(\vec{p}),\left(\sum_{i=1}^{n} b_{i 1} q_{i-1}, \ldots, \sum_{i=1}^{n} b_{i n} q_{i-1}\right)\right) \cdot\left[\begin{array}{ccc}
a_{11} & \ldots & a_{1 n} \\
\ldots & \ldots & \ldots \\
a_{m 1} & \ldots & a_{m n}
\end{array}\right]\right)= \\
& =\left(L(\vec{p}), a_{11} \sum_{i=1}^{n} b_{i 1} q_{i-1}+\ldots+a_{n 1} \sum_{i=1}^{n} b_{i n} q_{i-1}, \ldots, a_{1 n} \sum_{i=1}^{n} b_{i 1} q_{i-1}+\ldots+a_{n n} \sum_{i=1}^{n} b_{i n} q_{i-1}\right)
\end{aligned}
$$

The calculation of the right-hand side: We obtain

$$
\delta_{3}(\mathbf{A} \circ \mathbf{B},(L(\vec{p}), \vec{q})) \cup\left\{\delta_{1}\left(\mathcal{R}_{n}, L(\vec{p})\right) \times \delta_{3}\left(\mathbb{M}_{n, n}\left(\mathbb{R}^{+}\right), \vec{q}\right)\right\},
$$

which is a union of two sets. For easier reference we will treat both sets separately. 
First,

$$
\begin{gathered}
\delta_{3}(\mathbf{A} \circ \mathbf{B},(L(\vec{p}), \vec{q}))= \\
\left.=\delta_{3}\left(\mathbf{C}=\left\{c_{i j} ; c_{i j} \leq \min \left\{a_{i j}, b_{i j}\right\}, i, j \in \mathbb{N}_{n}\right\},(L \overrightarrow{(p}), \overrightarrow{(q)}\right)\right)= \\
=\left\{\left(L(\vec{p}),\left(\vec{q} \cdot \mathbf{C}=\left\{c_{i j} ; c_{i j} \leq \min \left\{a_{i j}, b_{i j}\right\}, i, j \in \mathbb{N}_{n}\right\}\right)\right\}=\right. \\
=\left\{\left(L(\vec{p}), c_{11} q_{0}+\cdots+c_{n 1} q_{n-1}, \cdots, c_{1 n} q_{0}+\cdots+c_{n n} q_{n-1}\right) ;\right. \\
\left.c_{i j} \leq \min \left\{a_{i j}, b_{i j}\right\}, i, j \in \mathbb{N}_{n}\right\} .
\end{gathered}
$$

We want to show that the pair on the left-hand side is an element of the set on the right-hand side. Since the first components are the same, we must concentrate on the second components. The left-hand side can be expanded and the first component of this vector rewritten to

$$
\begin{aligned}
q_{0}\left(a_{11} b_{11}+a_{21} b_{12}+\ldots+a_{n 1} b_{1 n}\right)+q_{1}\left(a_{11} b_{21}+a_{21} b_{22}\right. & \left.+\ldots+a_{n 1} b_{2 n}\right)+ \\
& +\ldots q_{n-1}(\ldots)
\end{aligned}
$$

Denote the sum in the $i$-th brackets as $S_{i}$. We must compare expression (20) and the sum

$$
c_{11} q_{0}+\cdots+c_{n 1} q_{n-1}
$$

It is obvious that on e.g. the sufficient condition that $a_{i j}, b_{i j} \geq 1$ for all $i, j \in \mathbb{N}_{n}$, i.e. considering $\mathbb{M}_{n, n}\left(\mathbb{R}_{1}^{+}\right)$instead of $\mathbb{M}_{n, n}\left(\mathbb{R}^{+}\right)$, expression (20) is an element of the principal end generated by element (21), i.e. is greater than or equal to element (20). The proof for other compoments is completely analogical.

Second,

$$
\begin{gathered}
\left\{\delta_{1}\left(\mathcal{R}_{n}, L(\vec{p})\right) \times \delta_{3}\left(\mathbb{M}_{n, n}\left(\mathbb{R}^{+}\right), \vec{q}\right)\right\}= \\
=\left\{\bigcup_{\vec{r} \in \mathcal{R}_{n}} \delta_{1}(\vec{r}, L(\vec{p})) \times \bigcup_{\mathbf{A} \in \mathbb{M}_{n, n}\left(\mathbb{R}^{+}\right)} \delta_{3}(\vec{q}, \mathbf{A})\right\}=\left\{(L(\vec{r} \vec{p}), \vec{\varphi}) ; \vec{r} \in \mathcal{R}_{n}, \vec{\varphi} \in \mathscr{R}_{n}^{\mathbb{F}}(\Omega)\right\} .
\end{gathered}
$$

For $\vec{r}=(1, \cdots, 1)$ and

$$
\vec{\varphi}=a_{11} \sum_{i=1}^{n} b_{i 1} q_{i-1}+\ldots+a_{n 1} \sum_{i=1}^{n} b_{i n} q_{i-1}, \ldots, a_{1 n} \sum_{i=1}^{n} b_{i 1} q_{i-1}+\ldots+a_{n n} \sum_{i=1}^{n} b_{i n} q_{i-1}
$$


we have

$$
\begin{aligned}
& \left\{\left(L(\vec{p}), c_{11} q_{0}+\cdots+c_{n 1} q_{n-1}, \cdots, c_{1 n} q_{0}+\cdots+c_{n n} q_{n-1}\right) ;\right. \\
& \left.\left.c_{i j} \leq \min \left\{a_{i j}, b_{i j}\right\}, i, j \in \mathbb{N}_{n}\right)\right\}=(L(\vec{r} \vec{p}), \vec{\varphi}) .
\end{aligned}
$$

Thus E-GMAC is satisfied even in cases when $a_{i j}, b_{i j} \in\langle 0,1)$ for some $i, j \in$ $\mathbb{N}_{n}$. (Negative values are not permitted by definition of $\mathbb{M}_{n, n}\left(\mathbb{R}^{+}\right)$.)

ad 3) In (19) we have: The calculation of the left hand side:

$$
\begin{gathered}
\left(\delta_{1} \cdot \delta_{3}\right)\left(\vec{r},\left(\delta_{1} \cdot \delta_{3}\right)(\mathbf{A},(L(\vec{p}), \vec{q}))\right)=\left(\delta_{1}\left(\vec{r},\left(\delta_{3}(\mathbf{A}, L(\vec{p})), \vec{q}\right)\right)\right)= \\
=\left(\delta_{1}(\vec{r},(L(\vec{p}), \vec{\varphi}))\right)=(L(\vec{r} \vec{p}), \vec{\varphi}) .
\end{gathered}
$$

The calculation of the right hand side:

$$
\begin{gathered}
\left(\delta_{1} \cdot \delta_{3}\right)(\vec{r} \triangle \mathbf{A},(L(\vec{p}), \vec{q})) \cup\left\{\delta_{1}\left(\mathcal{R}_{n}, L(\vec{p})\right) \times \delta_{3}\left(\mathbb{M}_{n, n}\left(\mathbb{R}^{+}\right), \vec{q}\right)\right\}= \\
=\left(\delta_{1} \cdot \delta_{3}\right)(\{\vec{r}, \mathbf{A}\},(L(\vec{p}), \vec{q})) \cup\left\{\delta_{1}\left(\mathcal{R}_{n}, L(\vec{p})\right) \times \delta_{3}\left(\mathbb{M}_{n, n}\left(\mathbb{R}^{+}\right), \vec{q}\right)\right\}= \\
=\delta_{1}(\vec{r},(L(\vec{p}), \vec{q})) \cup \delta_{3}(\mathbf{A}, L(\vec{p}), \vec{q}) \cup\left\{\bigcup_{\vec{r} \in \mathcal{R}_{n}} \delta_{1}(\vec{r}, L(\vec{p})) \times \bigcup_{\mathbf{A} \in \mathbb{M}_{n, n}\left(\mathbb{R}^{+}\right)} \delta_{3}(\vec{q}, \mathbf{A})\right\}= \\
=\left\{(L(\vec{r} \vec{p}), \vec{q}) ; \vec{r} \in \mathcal{R}_{n}\right\} \cup\left\{(L(\vec{p}), \vec{\varphi}) ; \vec{\varphi} \in \mathscr{R}_{n}^{\mathbb{F}}(\Omega)\right\} \\
\cup\left\{(L(\vec{r} \vec{p}), \vec{\varphi}) ; \vec{r} \in \mathcal{R}_{n}, \vec{\varphi} \in \mathscr{R}_{n}^{\mathbb{F}}(\Omega)\right\} .
\end{gathered}
$$

Thus the element $(L(\vec{r} \vec{p}), \vec{\varphi})$ on the left hand side is included in the extending set $\left\{(L(\vec{r} \vec{p}), \vec{\varphi}) ; \vec{r} \in \mathcal{R}_{n}, \vec{\varphi} \in \mathscr{R}_{n}^{\mathbb{F}}(\Omega)\right\}$ and the E-GMAC condition is satisfied in this case as well.

ad 4 In (19) we have: The calculation of the left hand side:

$$
\begin{gathered}
\left(\delta_{1} \cdot \delta_{3}\right)\left(\mathbf{A},\left(\delta_{1} \cdot \delta_{3}\right)(\vec{r},(L(\vec{p}), \vec{q}))\right)=\left(\delta_{3}\left(\mathbf{A},\left(\delta_{1}(\vec{r}, L(\vec{p})), \vec{q}\right)\right)\right)= \\
=\left(\delta_{3}(\mathbf{A},(L(\vec{r} \vec{p}), \vec{q}))\right)=(L(\vec{r} \vec{p}), \vec{\varphi}) .
\end{gathered}
$$

The calculation of the right hand side:

$$
\begin{gathered}
\left(\delta_{1} \cdot \delta_{3}\right)(\mathbf{A} \triangle \vec{r},(L(\vec{p}), \vec{q})) \cup\left\{\delta_{1}\left(\mathcal{R}_{n}, L(\vec{p})\right) \times \delta_{3}\left(\mathbb{M}_{n, n}\left(\mathbb{R}^{+}\right), \vec{q}\right)\right\}= \\
=\left(\delta_{1} \cdot \delta_{3}\right)(\{\mathbf{A}, \vec{r}\},(L(\vec{p}), \vec{q})) \cup\left\{\delta_{1}\left(\mathcal{R}_{n}, L(\vec{p})\right) \times \delta_{3}\left(\mathbb{M}_{n, n}\left(\mathbb{R}^{+}\right), \vec{q}\right)\right\}= \\
=\delta_{3}(\mathbf{A},(L(\vec{p}), \vec{q})) \cup \delta_{1}(\vec{r}, L(\vec{p}), \vec{q}) \cup\left\{\bigcup_{\vec{r} \in \mathcal{R}_{n}} \delta_{1}(\vec{r}, L(\vec{p})) \times \bigcup_{\mathbf{A} \in \mathbb{M}_{n, n}\left(\mathbb{R}^{+}\right)} \delta_{3}(\vec{q}, \mathbf{A})\right\}= \\
=\left\{(L(\vec{p}), \vec{\varphi}) ; \vec{\varphi} \in \mathscr{R}_{n}^{\mathbb{F}}(\Omega)\right\} \cup\left\{(L(\vec{r} \vec{p}), \vec{q}) ; \vec{r} \in \mathcal{R}_{n}\right\} \\
\cup\left\{(L(\vec{r} \vec{p}), \vec{\varphi}) ; \vec{r} \in \mathcal{R}_{n}, \vec{\varphi} \in \mathscr{R}_{n}^{\mathbb{F}}(\Omega)\right\} .
\end{gathered}
$$


Thus the element $(L(\vec{r} \vec{p}), \vec{\varphi})$ on the left hand side is included in the extending set $\left\{(L(\vec{r} \vec{p}), \vec{\varphi}) ; \vec{r} \in \mathcal{R}_{n}, \vec{\varphi} \in \mathscr{R}_{n}^{\mathbb{F}}(\Omega)\right\}$ and the E-GMAC condition is satisfied.

Since in all the possible cases the E-GMAC condition holds, the structure

$$
\left(\left(\mathcal{R}_{n} \cup \mathbb{M}_{n, n}\left(\mathbb{R}^{+}\right)\right), \mathbb{L}_{\mathbb{A}_{n}}(T) \times \mathscr{R}_{n}^{\mathbb{F}}(\Omega), \delta_{1} \cdot \delta_{3}\right)
$$

is a quasi-multiautomaton.

Remark 2. Notice that in case "ad 1)" of the above proof the usual GMAC condition may be used.

The following example is included in order to help understand the reasoning in part "ad 2)" of the proof of the above Theorem 5, especially the necessity of imposing the restrictions on coefficients of elements of $\mathbb{M}_{n, n}\left(\mathbb{R}^{+}\right)$. Compare this to the corresponding part of the proof of Theorem 6 .

Example 2. Suppose cartesian composition of e-quasi-multiautomata $\mathbb{A}_{1}$ and $\mathbb{A}_{3}$ with specific elements of inputs and states, where $L(\vec{p})=L\left(x^{2}, \mathrm{e}^{x}\right), \vec{q}=$ $(1,1)$ and $\mathbf{A}=\left[\begin{array}{ll}0.8 & 0.1 \\ 0.3 & 0.4\end{array}\right]$ and $\mathbf{B}=\left[\begin{array}{ll}0.7 & 0.1 \\ 0.1 & 0.3\end{array}\right]$. Then the E-GMAC condition (6) turns into

$$
\begin{aligned}
& \delta_{3}\left(\left[\begin{array}{cc}
0.8 & 0.1 \\
0.3 & 0.04
\end{array}\right], \delta_{3}\left(\left[\begin{array}{cc}
0.7 & 0.1 \\
0.1 & 0.3
\end{array}\right]\right.\right.\left.\left.,\left(L\left(x^{2}, \mathrm{e}^{x}\right),(1,1)\right)\right)\right) \in \\
& \in \delta_{3}\left(\left[\begin{array}{cc}
0.8 & 0.1 \\
0.3 & 0.04
\end{array}\right] \circ\left[\begin{array}{cc}
0.7 & 0.1 \\
0.1 & 0.3
\end{array}\right],\left(L\left(x^{2}, \mathrm{e}^{x}\right),(1,1)\right)\right) \cup \\
& \cup\left\{\delta_{1}\left(\mathcal{R}_{2}, L\left(x^{2}, \mathrm{e}^{x}\right)\right) \times \delta_{3}\left(\mathbb{M}_{2,2}\left(\mathbb{R}^{+}\right),(1,1)\right)\right\},
\end{aligned}
$$

i.e.

$$
\begin{aligned}
& \delta_{3}\left(L\left(x^{2}, \mathrm{e}^{x}\right),(\right. {\left.\left.\left[\begin{array}{cc}
0.8 & 0.1 \\
0.3 & 0.04
\end{array}\right],(0.8,0.4)\right)\right) \in } \\
& \in \delta_{3}\left(L\left(x^{2}, \mathrm{e}^{x}\right),\left(\left\{\left[\begin{array}{ll}
c_{11} & c_{12} \\
c_{21} & c_{22}
\end{array}\right] ; c_{11} \geq 0.7, c_{12} \geq 0.1, c_{21} \geq 0.1, c_{22} \geq 0.3\right\},(1,1)\right)\right) \cup \\
& \cup\left\{\left\{L\left(r_{0} x^{2}, r_{1} \mathrm{e}^{x}\right) ; r_{0}, r_{1} \in \mathcal{R}_{2}\right\} \times\left\{\left(\varphi_{0}, \varphi_{1}\right) ; \varphi_{0}, \varphi_{1} \in \mathscr{R}_{2}^{\mathbb{F}}(\Omega)\right\}\right\},
\end{aligned}
$$

i.e.

$$
\begin{aligned}
& \left(L\left(x^{2}, \mathrm{e}^{x}\right),(0.652,0.096)\right) \in \\
& \in\left\{\left(L\left(x^{2}, \mathrm{e}^{x}\right),\left(c_{11}+c_{21}, c_{12}+c_{22}\right)\right) ; c_{11}+c_{21} \geq 0.8, c_{12}+c_{22} \geq 0.4\right\} \cup \\
& \cup\left\{\left(L\left(r_{0} x^{2}, r_{1} \mathrm{e}^{x}\right),\left(\varphi_{0}, \varphi_{1}\right)\right) ; r_{0}, r_{1} \in \mathcal{R}_{2}, \varphi_{0}, \varphi_{1} \in \mathscr{R}_{2}^{\mathbb{F}}(\Omega)\right\} .
\end{aligned}
$$


In this last union of sets denote the first set $X$ and the second set $Y$. Condition E-GMAC (6) holds thanks to the set $Y$. In order to secure validity of the original GMAC condition (1), which is represented by the set $X$ only, we could impose the restriction $a_{i j}, b_{i j} \geq 1$ for all $i, j \in\{1,2\}$ on entries of matrices $\mathbf{A}, \mathbf{B} \in \mathbb{M}_{2,2}\left(\mathbb{R}^{+}\right)$. Notice that this condition is sufficient yet not necessary.

In the below Theorem 6 notice that "zero states" become relevant in part "ad 1)" of the proof in connection with $\delta_{2}(\vec{s}, \vec{q})$. The zero state of $\rho$ is the $n$-component vector $(0, \ldots, 0)$ while the zero state of $\mathbb{L} \mathbb{A}_{n}(T)$ is the operator $L(0, \ldots, 0)$ which corresponds to the differential equation $y^{(n)}(t)=0$.

Theorem 6. Consider se-quasi-multiautomata $\mathbb{A}_{1}$ and $\mathbb{A}_{2}$ constructed in Theorem 2 and Theorem 3 respectively. Define

$$
\left(\delta_{1} \cdot \delta_{2}\right):\left(\mathcal{R}_{n} \cup \mathbb{M}_{n, n}\left(\mathbb{R}_{1}^{+}\right)\right) \times\left(\mathbb{L}_{n}(T) \times \mathscr{R}_{n}^{\mathbb{F}}(\Omega)\right) \rightarrow \mathbb{L}_{n}(T) \times \mathscr{R}_{n}^{\mathbb{F}}(\Omega)
$$

by

$$
\left(\delta_{1} \cdot \delta_{2}\right)(X,(L(\vec{p}), \vec{q}))= \begin{cases}\left(\delta_{1}(X, L(\vec{p})), \vec{q}\right) & \text { if } X=\vec{r} \in \mathcal{R}_{n}, \\ \left(L(\vec{p}), \delta_{2}(X, \vec{q})\right) & \text { if } X=\mathbf{A} \in \mathbb{M}_{n, n}\left(\mathbb{R}_{1}^{+}\right), \\ \delta_{1}(X, L(\vec{p}))=0 & \text { if } X=\mathbf{A} \in \mathbb{M}_{n, n}\left(\mathbb{R}_{1}^{+}\right), \\ \delta_{2}(X, \vec{q})=0 & \text { if } X=\vec{r} \in \mathcal{R}_{n},\end{cases}
$$

Then the structure $\left(\left(\left(\mathcal{R}_{n} \cup \mathbb{M}_{n, n}\left(\mathbb{R}_{1}^{+}\right)\right), \triangle_{1}\right), \mathbb{L}_{\mathbb{A}_{n}}(T) \times \mathscr{R}_{n}^{\mathbb{F}}(\Omega), \delta_{1} \cdot \delta_{2}\right)$, where $\triangle_{1}$ is a restriction of $\triangle(15)$ on $\mathbb{M}_{n, n}\left(\mathbb{R}_{1}^{+}\right)$, is the cartesian composition $\mathbb{A}_{1} \cdot S E \mathbb{A}_{2}$.

Proof. We are going to show that the structure $\left(\left(\left(\mathcal{R}_{n} \cup \mathbb{M}_{n, n}\left(\mathbb{R}_{1}^{+}\right)\right), \triangle_{1}\right)\right.$, $\left.\mathbb{L}_{n}(T) \times \mathscr{R}_{n}^{\mathbb{F}}(\Omega), \delta_{1} \cdot \delta_{2}\right)$ satisfies the SE-GMAC condition. Notice that the proof follows the pattern of the proof of Theorem 5. In all cases 1) -4 ) the left-hand sides of the expressions are identical to the ones included in the proof of Theorem 5. For this reason we do not include them. Again, we have four cases:

1) $X=\vec{r} \in \mathcal{R}_{n}, Y=\vec{s} \in \mathcal{R}_{n}$,

2) $X=\mathbf{A} \in \mathbb{M}_{n, n}\left(\mathbb{R}_{1}^{+}\right), Y=\mathbf{B} \in \mathbb{M}_{n, n}\left(\mathbb{R}_{1}^{+}\right)$,

3) $X=\vec{r} \in \mathcal{R}_{n}, Y=\mathbf{A} \in \mathbb{M}_{n, n}\left(\mathbb{R}_{1}^{+}\right)$,

4) $X=\mathbf{A} \in \mathbb{M}_{n, n}\left(\mathbb{R}_{1}^{+}\right), Y=\vec{r} \in \mathcal{R}_{n}$.

We show that the SE-GMAG condition

$$
\begin{aligned}
\left(\delta_{1} \cdot \delta_{2}\right)\left(X,\left(\delta_{1} \cdot \delta_{2}\right)(Y,(L(\vec{p}), \vec{q}))\right) \in\left(\delta_{1} \cdot \delta_{2}\right)\left(X \triangle_{1} Y,(L(\vec{p}), \vec{q})\right) \cup \\
\cup\left\{\left(\delta_{1}(X, L(\vec{p})), \delta_{2}(Y, \vec{q})\right),\left(\delta_{1}(Y, L(\vec{p})), \delta_{2}(X, \vec{q})\right)\right\}
\end{aligned}
$$


is valid for all four cases. For easier manipulation we define

$$
\vec{q} \cdot\left[\begin{array}{ccc}
a_{11} & \ldots & a_{1 n} \\
\ldots & \ldots & \ldots \\
a_{n 1} & \ldots & a_{n n}
\end{array}\right]=\vec{\varphi} .
$$

ad 1) In (23) we have: For the calculation of the left hand side see proof of Theorem 5. The calculation of the right hand side:

$$
\begin{aligned}
& \delta_{1}(\vec{r} \bullet \vec{s},(L(\vec{p}), \vec{q})) \cup\left\{\left(\delta_{1}(\vec{r}, L(\vec{p})), \delta_{2}(\vec{s}, \vec{q})\right),\left(\delta_{1}(\vec{s}, L(\vec{p})), \delta_{2}(\vec{r}, \vec{q})\right)\right\}= \\
= & \bigcup_{\vec{t} \geq \vec{r} \vec{s}}\left(\delta_{1}(\vec{t}, L(\vec{p})), \vec{q}\right) \cup\{(L(\vec{r} \vec{p}), 0),(L(\vec{s} \vec{p}), 0)\}= \\
= & \{(L(\vec{t} \vec{p}), \vec{q}) ; \vec{t} \geq \vec{r} \vec{s}\} \cup\{(L(\vec{r} \vec{p}), 0),(L(\vec{s} \vec{p}), 0)\} .
\end{aligned}
$$

For $\vec{t}=\vec{r} \vec{s}$ we have

$$
\left(L\left(r_{0} s_{0} p_{0}, \ldots, r_{n-1} s_{n-1} p_{n-1}\right), \vec{q}\right)=\left(L\left(t_{0} p_{0}, \ldots, t_{n-1} p_{n-1}\right), \vec{q}\right),
$$

where $t \in \mathcal{R}_{n}$. Thus

$\left(L\left(r_{0} s_{0} p_{0}, \ldots, r_{n-1} s_{n-1} p_{n-1}\right), \vec{q}\right) \in\{(L(\vec{t} \vec{p}), \vec{q}) ; \vec{t} \geq \vec{r} \vec{s}\} \cup\{(L(\vec{r} \vec{p}), 0),(L(\vec{s} \vec{p}), 0)\}$

and SE-GMAC holds.

ad 2) In (23) we have: For the calculation of the left hand side see proof of Theorem 5. The calculation of the right hand side:

$$
\begin{gathered}
\left(\delta_{1} \cdot \delta_{2}\right)(\mathbf{A} \triangle \mathbf{B},(L(\vec{p}), \vec{q})) \cup\left\{\left(\delta_{1}(\mathbf{A}, L(\vec{p})), \delta_{2}(\mathbf{B}, \vec{q})\right),\left(\delta_{1}(\mathbf{B}, L(\vec{p})), \delta_{2}(\mathbf{A}, \vec{q})\right)\right\}= \\
=\delta_{2}(\mathbf{A} \triangle \mathbf{B},(L(\vec{p}), \vec{q})) \cup\left\{\left(0, \delta_{2}(\mathbf{A}, \vec{q})\right),\left(0, \delta_{2}(\mathbf{B}, \vec{q})\right)\right\}= \\
=\left\{(L(\vec{p}), \vec{q} \cdot \mathbf{C}) ; \mathbf{C}=\left(c_{i j}\right) ; c_{i j} \geq \min \left\{a_{i j}, b_{i j}\right\}, i, j \in \mathbb{N}_{n}\right\} \cup \\
\cup\left\{\left(0, \delta_{2}(\mathbf{A}, \vec{q})\right),\left(0, \delta_{2}(\mathbf{A}, \vec{q})\right)\right\}
\end{gathered}
$$

At this point recall the proof of Theorem 5, part "ad 2)". Again, we have a union of two sets. However, the element on the left hand side can never belong to the second set. Therefore, the condition SE-GMAC can be satisfied only if the element on the left hand side belongs to

$$
\left\{(L(\vec{p}), \vec{q} \cdot \mathbf{C}) ; \mathbf{C}=\left(c_{i j}\right) ; c_{i j} \geq \min \left\{a_{i j}, b_{i j}\right\}, i, j \in \mathbb{N}_{n}\right\} .
$$

However, as has already been shown in the proof of Theorem 5, part "ad 2)", this holds e.g. on the sufficient condition $a_{i j}, b_{i j} \geq 1$ for all $i, j \in \mathbb{N}_{n}$. 
ad 3) In (23) we have: For the calculation of the left hand side see the proof of Theorem 5. The calculation of the right hand side:

$$
\begin{gathered}
\left(\delta_{1} \cdot \delta_{2}\right)(\vec{r} \triangle \mathbf{A},(L(\vec{p}), \vec{q})) \cup\left\{\left(\delta_{1}(\vec{r}, L(\vec{p})), \delta_{2}(\mathbf{A}, \vec{q})\right),\left(\delta_{1}(\mathbf{A}, L(\vec{p})), \delta_{2}(\vec{r}, \vec{q})\right)\right\}= \\
=\left(\delta_{1} \cdot \delta_{2}\right)(\{\vec{r}, \mathbf{A}\},(L(\vec{p}), \vec{q})) \cup\{(L(\vec{r} \vec{p}), \vec{\varphi}),(0,0)\}= \\
=\delta_{1}(\vec{r},(L(\vec{p}), \vec{q})) \cup \delta_{2}(\mathbf{A},(L(\vec{p}), \vec{q})) \cup\{(L(\vec{r} \vec{p}), \vec{\varphi}),(0,0)\}= \\
=\left\{(L(\vec{r} \vec{p}), \vec{q}) ; \vec{r} \in \mathcal{R}_{n}\right\} \cup\left\{(L(\vec{p}), \vec{\varphi}) ; \vec{\varphi} \in \mathscr{R}_{n}^{\mathbb{F}}(\Omega)\right\} \cup\{(L(\vec{r} \vec{p}), \vec{\varphi}),(0,0)\} .
\end{gathered}
$$

Thus the element $(L(\vec{r} \vec{p}), \vec{\varphi})$ of the left hand side is directly included in the extending set $\{(L(\vec{r} \vec{p}), \vec{\varphi}),(0,0)\}$ and the SE-GMAC condition holds in this case.

ad 4) In (23) we have: For the calculation of the left hand side see proof of Theorem 5. The calculation of the right hand side:

$$
\begin{gathered}
\left(\delta_{1} \cdot \delta_{2}\right)(\{\mathbf{A}, \vec{r}\},(L(\vec{p}), \vec{q})) \cup\left\{\left(\delta_{1}(\mathbf{A}, L(\vec{p})), \delta_{2}(\vec{r}, \vec{q})\right),\left(\delta_{1}(\vec{r}, L(\vec{p})), \delta_{2}(\mathbf{A}, \vec{q})\right)\right\}= \\
=\delta_{2}(\mathbf{A},(L(\vec{p}), \vec{q})) \cup \delta_{1}((\vec{r}, L(\vec{p})), \vec{q}) \cup\{(0,0),(L(\vec{r} \vec{p}), \vec{\varphi})\}= \\
=\left\{(L(\vec{p}), \vec{\varphi}) ; \vec{\varphi} \in \mathscr{R}_{n}^{\mathbb{F}}(\Omega)\right\} \cup\left\{(L(\vec{r} \vec{p}), \vec{q}) ; \vec{r} \in \mathcal{R}_{n}\right\} \cup\{(0,0),(L(\vec{r} \vec{p}), \vec{\varphi})\} .
\end{gathered}
$$

Thus the element $(L(\vec{r} \vec{p}), \vec{\varphi})$ of the left hand side is also included in the extending set $\{(0,0),(L(\vec{r} \vec{p}), \vec{\varphi})\}$ and the SE-GMAC condition holds in this case.

Since for all the possible cases the SE-GMAC condition holds, the structure

$$
\left(\left(\mathcal{R}_{n} \cup \mathbb{M}_{n, n}\left(\mathbb{R}_{1}^{+}\right)\right), \mathbb{L}_{\mathbb{A}_{n}}(T) \times \mathscr{R}_{n}^{\mathbb{F}}(\Omega), \delta_{1} \cdot \delta_{2}\right)
$$

is a quasi-multiautomaton.

Example 3. Example 1 shows that the original GMAC condition (16) is not sufficient to construct the cartesian composition of quasi-multiautomata $\mathbb{A}_{1}$ and $\mathbb{A}_{2}$. However, if we considered the SE-GMAC condition (7) for the context of Example 1, the right-hand side of computation (17) of the example would be extended by

$$
\begin{aligned}
\cup\left\{\left(\delta_{1}\left(\left[\begin{array}{ll}
2 & 4 \\
3 & 1
\end{array}\right], L\left(x^{2}, x\right)\right)\right.\right. & \left., \delta_{3}((1,2),(x, 0))\right), \\
& \left.\left(\delta_{1}\left((1,2), L\left(x^{2}, x\right)\right), \delta_{3}\left(\left[\begin{array}{ll}
2 & 4 \\
3 & 1
\end{array}\right],(x, 0)\right)\right)\right\},
\end{aligned}
$$

i.e. united with $\left\{(0,0),\left(L\left(x^{2}, 2 x\right),(2 x, 4 x)\right)\right\}$, which would provide that the condition would hold. 
Remark 3. The concept of the cartesian composition of automata - introduced by Dörfler [8] as a new product of automata - is investigated in [8] with respect to connectedness, commutativity, perfectness, endomorphism transformations, congruences, cyclicity and to other important properties. Studying these properties in the context of quasi-multiautomata and the cartesian composition performed using E-GMAC or SE-GMAC would be an interesting line of research as it is obvious that especially thanks to the inclusion of the zero state properties of $\mathbb{A}_{i} \cdot{ }_{E} \mathbb{A}_{j}$ and $\mathbb{A}_{i} \cdot S E \mathbb{A}_{j}$ will differ. It is also to be noted that one must distinguish the commutativity of the composition in the sense of Dörfler [8], i.e. $\mathbb{A}_{i} \cdot \mathbb{A}_{j}=\mathbb{A}_{j} \cdot \mathbb{A}_{i}$, and commutativity with respect to the GMAC conditions, i.e. $\delta(s, x \cdot y)=\delta(s, y \cdot x)$.

\section{References}

[1] A. R. Ashrafi, A. Madanshekaf, Generalized action of a hypergroup on a set, Italian J. Pure and Appl. Math., 15(3) (1998), 127-135.

[2] R. A. Borzooei, H. R. Varasteh, A. Hasankhani, F-Multiautomata on join spaces induced by differential operators, Appl. Math. (Irvine) 5 (2014), 1386-1391.

[3] J. Chvalina, Infinite multiautomata with phase hypergroups of various operators. In Proc. 10th International Congress on Algebraic Hyperstructures and Applications; Hošková, Š., Ed.; University of Defense: Brno, 2009 .

[4] J. Chvalina, L. Chvalinová, Join space of linear ordinary differential operators of the second order, Folia Fac. Sci. Nat. Univ. Masarykianae Brunesis, Mathematica 13 (2002), 77-86 (Colloqium on Differential and Difference Equations, CDDE 2002).

[5] J. Chvalina, Š. Hošková-Mayerová, On certain proximities and preorderings on the transposition hypergroups of linear first-order partial differential operators, An. St. Univ. Ovidius Constanţa, 22(1) (2014), 85-103.

[6] J. Chvalina, Š. Hošková-Mayerová, A. Dehghan Nezhad, General actions of hyperstructures and some applications, An. Şt. Univ. Ovidius Constanţa, 21(1) (2013), 59-82.

[7] P. Corsini, V. Leoreanu, Applications of Hyperstructure Theory, Kluwer Academic Publishers, Dordrecht, 2003.

[8] W. Dörfler, The cartesian composition of automata, Math. System Theory 11 (1978), 239-257. 
[9] F. Gécseg, I. Peák, Algebraic Theory of Automata, Budapest, Akadémia Kiadó, 1972.

[10] D. S. Malik, J. N. Mordenson, M. K. Sen, The cartesian composition of fuzzy finite state machines, Kybernetics, 24 (1995), 98-110.

[11] G. G. Massouros, Hypercompositional structures in the theory of languages and automata. An. Şt. Univ. A.I. Çuza Iaşi, Sect. Inform., III (1994), 65-73.

[12] G. G. Massouros, J. Mittas, Languages, Automata and Hypercompositional Structures, In Proceedings of the 4th International Congress on Algebraic Hyperstructures and Applications, Xanthi 1990; World Scientific, 1991.

[13] J. N. Mordenson, D. S. Malik, Fuzzy Automata and Languages - Theory and Applications, Chapman \& Hall CRC Press, 2002.

[14] M. Novák, Some basic properties of EL-hyperstructures, European J. Combin., 34 (2013), 446-459.

[15] M. Novák, On EL-semihypergroups, European J. Combin. 44 Part B (2015), 274-286.

[16] S. Subrmaniyan, M. Rajasekar, Cartesian composition in bipolar fuzzy finite state machines, International Journal of Computer Applications 92(11) (2014), 1-7.

[17] J. Zhan, S. Sh. Mousavi, J. Jafarpour, On hyperactions of hypergroups. U.P.B. Sci. Bull., Series A, 73(1) (2011), 117-128.

Jan CHVALINA,

Department of Mathematics,

Faculty of Electrical Engineering and Communication,

Brno University of Technology,

Technická 8, 61600 Brno, Czech Republic.

Email: chvalina@feec.vutbr.cz

Štěpán KŘEHLÍK,

Department of Mathematics,

Faculty of Electrical Engineering and Communication,

Brno University of Technology,

Technická 8, 61600 Brno, Czech Republic.

Email: stepan.krehlik@gmail.com 
CARTESian COMPosition and the PROBlem of generalizing the MAC CONDItion TO QUASI-MULTIAUTOMATA

Michal NOVÁK,

Department of Mathematics,

Faculty of Electrical Engineering and Communication,

Brno University of Technology,

Technická 8, 61600 Brno, Czech Republic.

Email: novakm@feec.vutbr.cz 